\title{
Patterns and Prognostic Value of Lymph Node Metastasis on Distant Metastasis and Survival in Nasopharyngeal Carcinoma: A Surveillance, Epidemiology, and End Results Study, 2006-2015
}

\author{
Yali Xu, ${ }^{1}$ Taoyuan Huang, ${ }^{2}$ Liqin Fan, ${ }^{1}$ Wei Jin, ${ }^{1}$ Xiaoming Chen, ${ }^{1}$ and Jinhai Chen $\mathbb{D}^{1}$ \\ ${ }^{1}$ Department of Otolaryngology, The Second Affiliated Hospital of Guangzhou Medical University, Guangzhou 510260, China \\ ${ }^{2}$ Department of Dermatology, Dermatology Hospital of Southern Medical University, Guangzhou 510091, China
}

Correspondence should be addressed to Jinhai Chen; ent_hai@163.com

Received 28 May 2019; Revised 25 July 2019; Accepted 22 August 2019; Published 29 November 2019

Academic Editor: Francesca De Felice

Copyright ( 2019 Yali Xu et al. This is an open access article distributed under the Creative Commons Attribution License, which permits unrestricted use, distribution, and reproduction in any medium, provided the original work is properly cited.

\begin{abstract}
This study was conducted to identify factors associated with lymph node (LN) metastasis in nasopharyngeal carcinoma (NPC) patients, analyze node distribution patterns, and explore the prognostic value of the LN metastasis level for survival. We included 2994 patients with primary NPC diagnosed between 2006 and 2015 with information in the Surveillance, Epidemiology, and End Results (SEER) database. Patients' demographic and clinicopathologic features were compared according to LN status using chisquared tests. The 5-year overall survival (OS) and cancer-specific survival (CSS) rates were calculated by the Kaplan-Meier method, and the differences were estimated by log-rank tests. Multivariate Cox proportional hazard models were used to evaluate independent risk factors for OS and CSS. Logistic regression was used to evaluate the risk of each LN metastasis category for distant metastasis. There were 695 patients in the N0 stage and 2299 with LN metastasis (classified as stage N1, N2, or N3). The overall incidence of LN metastasis was $76.8 \%$. Sex and T stage were not associated with LN metastasis. Older patients had a significantly worse 5-year OS and CSS than younger patients. In terms of histologic type, keratinizing squamous cell carcinoma had the lowest 5-year OS and CSS at $48.2 \%$ and 53.8\%, respectively. The most common nodal involvement level was II (65.9\%), followed by III (29.1\%), V (25.6\%), I (17.6\%), IV (15.7\%), and retropharynx (13.5\%). The skip metastasis rate was 5.7\% (130/2299). Patients with only level II metastasis (classified as level 2) was the most common category, accounting for $30 \%$. Compared to level 2, patients with only level I (classified as level 1) had an OR of 2.101 (95\% CI: 1.090-4.047, $P=0.027$ ) for distant metastasis, patients with simultaneous levels II, III, IV, and V (classified as levels 2345) had the highest OR of 4.064 (95\% CI: 2.155-7.666, $P<0.001$ ) for distant metastasis, and level 24 had an OR of 3.003 (95\% CI: 1.074-8.395, $P=0.036$ ) for distant metastasis. In survival analysis, levels 235 had a significant HR of 1.708 (95\% CI: 1.089-2.678, $P=0.020$ ) for CSS compared to level 2 after adjustment for age, sex, race, histology, TNM (tumor, node, and metastasis) stage, and treatment.
\end{abstract}

\section{Introduction}

Nasopharyngeal carcinoma (NPC) is a rare cancer arising from the nasopharynx epithelium. Globally, the incidence rate of NPC is less than 1 case per 100,000 person-years [1]; however, it is as high as 20 to 40 per 100,000 persons in endemic regions such as Southeast Asia and North Africa [2, 3].

Among head and neck cancers, NPC has the highest preponderance for regional lymph node (LN) metastasis [4]. Due to the region's vast submucosal avalvular lymph capillary network, the incidence of LN metastasis is as high as $70 \%$. With the development of advanced imaging methods such as magnetic resonance imaging (MRI) and positron emission tomography/computed tomography (PET/CT), LN metastasis is detected more easily and accurately. LN metastasis patterns are significant predictors of distant metastasis and have prognostic effects on overall and distant metastasis-free survival $[5,6]$. To our knowledge, most studies have focused on the distribution probabilities in each level [7-9]; however, there is limited information 
about prognostic value of LN metastasis category in distant metastasis and survival.

Radiotherapy is the primary and only curative treatment for NPC. Intensity-modulated radiotherapy (IMRT) is the preferred method as it provides the therapeutic benefit of dose escalation to tumor tissue with reduced toxicity to normal tissues [10]. Compared to other treatments, IMRT has reduced radiation-related toxicity and can improve NPC patient-reported quality of life [11]. Morbidities of IMRT including dermatitis, odynophagia, neck fibrosis, and brachial plexopathy are associated with the larger field of the cervical LN target volumes [12]. The definition of the appropriate clinical target volume for cervical LN in NPC is still a matter of debate [13].

The Surveillance, Epidemiology, and End Results (SEER) program is a well-established and extensive collection of data from cancer registries throughout the United States [14]. It includes almost all cancer incidence, prevalence, demographic, and clinicopathologic datasets. In this study, we sought to investigate factors associated with the incidence of LN metastasis and calculate hazard ratios (HRs), overall survival (OS), and cancer-specific survival (CSS) in 2994 NPC patients. We also analyzed LN distribution patterns and evaluated the LN metastasis categories, which may predict the prognosis of 1884 NPC patients with complete information of LN involvement level.

\section{Materials and Methods}

2.1. Study Population. The SEER program collects data from 18 original registries that cover approximately $27.8 \%$ of the U.S. population. We used SEER $*$ Stat version 8.3 .5 software to extract the data from the SEER database. We included patients who were diagnosed with only primary NPC from 2005 to 2016 using the International Classification of Diseases O-3 site recodes $\mathrm{C} 11.0-\mathrm{C} 11.9$ and histologic types 8020, 8021, 8070-8073, 8082, and 8083. Patients were excluded if the type of reporting source was from autopsy or death certificate. We also excluded those without complete stage information. Ultimately, 2994 patients were included in this study.

Incidence rates were age-adjusted to the standard U.S. 2000 population. The annual percent change (APC) in incidence was also calculated with a 1-year end-point, and significance testing was performed with weighted least squares.

Age was grouped into five categories: <40, 40-49, 50-59, 60-69, and $>70$ years. Racial classifications were Asian/Pacific Islander, White, Black, American Indian/Alaska Native, and unknown. Based on the World Health Organization (WHO) classification scheme, histology was divided into keratinizing squamous cell carcinoma (KSCC, SEER codes 8070, 8071; squamous cell carcinoma), nonkeratinizing carcinoma (NKSCC, SEER codes 8020, 8021, 8072, 8073, and 8082; undifferentiated, anaplastic, large- and small-cell nonkeratinizing carcinomas, and lymphoepithelial carcinoma), and basaloid squamous cell carcinoma (BSCC, SEER code 8083; basaloid squamous cell carcinoma). The AJCC 6th (diagnosis years from 2006 to 2009) and 7th (diagnosis years from 2010 to 2016) editions were used for staging. Based on a study that demonstrated superiority of the 7 th edition [15], we reclassified the T2a category in the 6th edition to T1, and T2b to T2. LN metastasis levels were coded based on the Collaborative Stage Site-Specific Factors (CS Site-Specific Factors 3 and 4) including levels I, II, III, IV, V, and retropharyngeal (rp) LNs. A patient with only level I metastasis was classified as level 1, the patient with level I and II was classified as levels 12 , etc. The patient that skipped a level would be classified accordingly (e.g., levels 124).

2.2. Statistical Analysis. Comparisons of categorical variables between patients with and without LN metastasis were performed using chi-squared tests. The 5-year OS rates and CSS rates were calculated by Kaplan-Meier analysis, and survival differences were estimated with log-rank tests. Logistic regression was used to evaluate the risk LN metastasis category for distant metastasis. We calculated HRs and their 95\% confidence intervals (CIs) using multivariate Cox proportional hazards regression models to evaluate the effects of LN metastasis compound category on CSS. All analyses were conducted using SPSS version 25 statistical software (IBM Corporation). $P<0.05$ was considered statistically significant.

\section{Results}

3.1. Incidence Analysis. We collected age-adjusted incidence data from the original 18 registry sites in the SEER database. The overall incidence of NPC during 2006-2015 was 0.33 patient per 100,000 population $(0.49 / 100,000$ for males and $0.19 / 100,000$ for females). The annual overall incidence rates for the study period are shown in Figure 1. They remained stable (APC 1.45, 95\% CI: -0.59-3.53, $P=0.14$ ) from 2005 to 2016.

3.2. Patient Baseline Characteristics. A total of 2994 NPC patients diagnosed between 2006 and 2015 were enrolled, including 2139 (71.4\%) males and 855 (28.6\%) females. The male-to-female ratio was $2.5: 1$. The median age at diagnosis was 54 (range, 8-99) years. Most patients were White (47.4\%), followed by Asian/Pacific Islander (37.7\%), Black (12.4\%), American Indian/Alaska Native (1.7\%), and unknown (0.8\%). Among NPC subtypes, NKSCC had the highest incidence at 55.6\%, compared with KSCC (43.0\%) and BSCC (1.4\%). As for the TNM stage, $1610(53.8 \%)$ patients were $\mathrm{T} 1+\mathrm{T} 2,634(21.2 \%)$ were $\mathrm{T} 3$, and $750(25.1 \%)$ were $\mathrm{T} 4 ; 1684(56.2 \%)$ were $\mathrm{N} 0+\mathrm{N} 1,906(30.3 \%)$ were $\mathrm{N} 2$, and $404(13.5 \%)$ were N3; $2695(90.0 \%)$ were M0, and 299 (10.0\%) were M1.

\subsection{Factors Associated with LN Metastasis. Correlations} between NPC patient characteristics and LN metastasis status are shown in Table 1. Younger patients (age at diagnosis $<40$ years) had a significantly higher percentage of LN metastasis than older patients ( $85.6 \%$ vs. $82.4 \%, 76.9 \%$, $71.3 \%$ for $40-49,50-59,60-69$, and $>70$ years, respectively, 

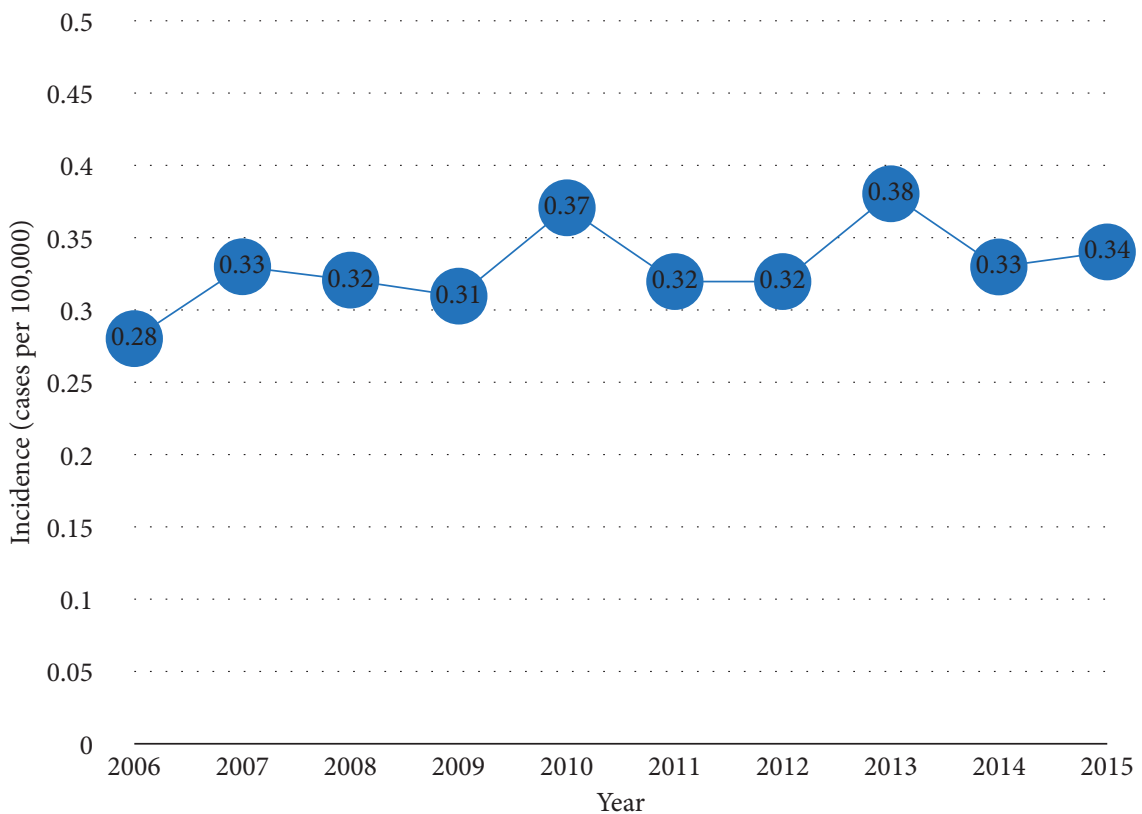

FIGURE 1: Incidence trends for NPC, 2006-2015.

TABLE 1: Comparison of clinicopathologic demographics between 2994 NPC patients according to LN involvement.

\begin{tabular}{|c|c|c|c|c|}
\hline \multirow{2}{*}{ Characteristics } & \multicolumn{3}{|c|}{$\mathrm{LN}$ involvement $\mathrm{a}^{\mathrm{a}}$} & \multirow{2}{*}{$P$} \\
\hline & N0 $(n, \%)$ & $\mathrm{N} 1-3(n, \%)$ & Total number & \\
\hline \multicolumn{5}{|l|}{ Age group (years) } \\
\hline$<40$ & $66(14.4)$ & $393(85.6)$ & 459 & $<0.001$ \\
\hline $40-49$ & $113(17.6)$ & $528(82.4)$ & 641 & \\
\hline $50-59$ & $204(23.1)$ & $681(76.9)$ & 885 & \\
\hline $60-69$ & $173(28.7)$ & $429(71.3)$ & 602 & \\
\hline$>70$ & $139(34.2)$ & $268(65.8)$ & 407 & \\
\hline \multicolumn{5}{|l|}{ Sex } \\
\hline Male & $491(23.0)$ & $1648(77.0)$ & 2139 & 0.596 \\
\hline Female & $204(23.9)$ & $651(76.1)$ & 855 & \\
\hline \multicolumn{5}{|l|}{ Race $^{b}$} \\
\hline Asian/Pacific Islander & $223(19.8)$ & $905(80.2)$ & 1128 & 0.002 \\
\hline White & $369(26.0)$ & $1051(74.0)$ & 1420 & \\
\hline Black & $83(22.4)$ & $287(77.6)$ & 370 & \\
\hline American Indian/Alaska Native & $9(17.3)$ & $43(82.7)$ & 52 & \\
\hline \multicolumn{5}{|l|}{ Histology } \\
\hline KSCC & $320(24.9)$ & $966(75.1)$ & 1286 & 0.004 \\
\hline NKSCC & $358(21.5)$ & $1307(78.5)$ & 1665 & \\
\hline BSCC & $17(39.5)$ & $26(60.5)$ & 43 & \\
\hline \multicolumn{5}{|l|}{ T stage ${ }^{c}$} \\
\hline $\mathrm{T} 1+\mathrm{T} 2$ & $363(22.5)$ & $1247(77.5)$ & 1610 & 0.479 \\
\hline T3 & $146(23.0)$ & $488(77.0)$ & 634 & \\
\hline $\mathrm{T} 4$ & $186(24.8)$ & $564(75.2)$ & 750 & \\
\hline \multicolumn{5}{|l|}{ M stage } \\
\hline M0 & $655(24.3)$ & $2040(75.7)$ & 2695 & $<0.001$ \\
\hline M1 & $40(13.4)$ & $259(86.6)$ & 299 & \\
\hline
\end{tabular}

BSCC, basaloid squamous cell carcinoma; LN, lymph node; KSCC, keratinizing squamous cell carcinoma; M, distant metastasis; N, node; NKSCC, nonkeratinizing carcinoma; $\mathrm{T}$, tumor. ${ }^{\mathrm{a}} \mathrm{N} 0$, none; $\mathrm{N} 1$, unilateral cervical and/or unilateral or bilateral retropharyngeal node(s), $\leq 6 \mathrm{~cm}$ in greatest dimension, above supraclavicular fossa; N2, bilateral cervical node(s), $\leq 6 \mathrm{~cm}$ in greatest dimension, above supraclavicular fossa; N3, N3a: $>6 \mathrm{~cm}$ in greatest dimension, above supraclavicular fossa; N3b: in supraclavicular fossa. ${ }^{\mathrm{b}} 24$ cases were missing. ${ }^{\mathrm{C}} \mathrm{T} 1$, nasopharynx, oropharynx, or nasal cavity; $\mathrm{T} 2$, parapharyngeal extension; T3, bony structures and/or paranasal sinuses; T4, intracranial extension and/or cranial nerves, hypopharynx, orbit, or infratemporal fossa/masticatory space.

$P<0.001)$. With regard to race, the American Indian/Alaska Native group had the highest rate of LN metastasis $(82.7 \%$ vs. $80.2 \%, 74.0 \%$, and $77.6 \%$ in Asian/Pacific Islander,
White, Black, and American Indian/Alaska Native, respectively, $P=0.002)$. LN metastasis was less common in patients with BSCC compared to other subtypes $(60.5 \%$ vs. 
$75.1 \%$ and $78.5 \%$ for the KSCC and NKSCC subtypes, respectively, $P=0.004$ ). Surprisingly, no significant correlation was found for the incidence of $\mathrm{LN}$ metastasis regarding sex or T stage (both $P>0.05$ ).

3.4. Influence of N Stage on 5-Year OS and CSS Rates and Multivariate Analysis Findings. The 5-year OS and CSS rates for the entire population were $59.4 \%$ and $63.7 \%$, respectively. As shown in Table 2, the 5-year OS and CSS were significantly longer in the $\mathrm{N} 0+\mathrm{N} 1$ group than in other groups (OS: $62.8 \%$ vs. $57.5 \%$ and $47.4 \%$ for $\mathrm{N} 2$ and N3 stages, respectively, $P<0.001$; CSS: $67.7 \%$ vs. $60.9 \%$ and $51.1 \%$ for N2 and N3 stages, respectively, $P<0.001)$. Younger patients (<40 years) had significantly longer OS and CSS than older patients (OS: $76.6 \%$ vs. $67.5 \%, 59.9 \%, 49.8 \%$, and $36.1 \%$ for $40-49,50-59,60-69$, and $>70$ years, respectively, $P<0.001$; CSS: $78.5 \%$ vs. $68.8 \%, 63.8 \%, 54.4 \%, 47.4 \%$ for $40-49,50-59$, $60-69$, and $>70$ years, respectively, $P<0.001)$. Female patients had significantly longer OS and CSS than male patients (OS: $64.1 \%$ vs. $57.6 \%, P=0.002$; CSS: $68.9 \%$ vs. $61.7 \%)$. In terms of race, after excluding patients categorized as unknown $(n=24)$, Asian/Pacific Islanders had the highest OS and CSS rates at $67.3 \%$ and $70.2 \%$, respectively, while American Indian/Alaska Native had the lowest OS and CSS at $43.8 \%$ and $45.3 \%$, respectively. Among histologic subtypes, KSCC had the lowest OS and CSS (OS: $48.2 \%$ vs. $67.9 \%$ and $61.8 \%$ in NKSCC and BSCC, respectively, $P<0.001$; CSS: $53.8 \%$ vs. $71.1 \%$ and $66.8 \%$ in NKSCC and BSCC, respectively, $P<0.001)$. Patients recommended to undergo surgery had better 5-year OS and CSS rates than those not/unknown recommended (OS: $65.9 \%$ vs. $58.2 \%$, $P=0.006$; CSS: $71.5 \%$ vs. $62.3 \%, P=0.004)$. A total of 846 patients received radiotherapy, and their 5-year OS and CSS were better than that in patients without radiotherapy (OS: $66.9 \%$ vs. $56.3 \%, P<0.001$; CSS: $71.8 \%$ vs. $60.4 \%, P<0.001)$. Finally, patients undergoing chemotherapy had higher 5year OS and CSS rates than those without chemotherapy (OS: $62.1 \%$ vs. $45.6 \%, P<0.001$; CSS: $65.9 \%$ vs. $52.4 \%$, $P<0.001)$. We performed multivariate analysis using Cox proportional hazards regression modeling and found that age, sex, race, histology type, TNM stage, surgery, radiotherapy, and chemotherapy were significant independent prognostic factors for OS and CSS. Compared with the $\mathrm{N} 0+\mathrm{N} 1$ group, N2 patients had a HR of 1.311 (95\% CI: $1.135-1.514, P<0.001)$ for OS and the N3 group had a HR of 1.625 (95\% CI:1.357-1.945, $P<0.01$ ) for OS. In addition, N2 patients had a HR of 1.351 for CSS (95\% CI: 1.156-1.580, $P<0.001)$ and the N3 group had a HR of 1.630 for CSS $(95 \%$ CI:1.342-1.979, $P<0.01)$.

3.5. Incidence and Distribution of LN Metastasis. A total of 2299 (76.8\%) patients with lymphadenopathy (classified as N1, N2, or N3) were enrolled. The distributions were as follows: 404 (17.6\%) cases in level I, 1515 (65.9\%) in level II, $670(29.1 \%)$ in level III, 360 (15.7\%) in level IV, 588 (25.6\%) in level V, and 311 (13.5\%) in the rp level. Eighty-one cases presented with level III LN involvement without level II LN involvement. We also identified 49 cases with level IV LN involvement without level II and III involvement. The skip metastasis rate was 5.7\% (130/2299).

3.6. The Risk LN Metastasis Categories for Distant Metastasis. After excluding 415 patients with incomplete LN metastasis level information, 1884 patients were ultimately unrolled. Level 2 is the most commonly-seen LN metastasis category in NPC patients with LN involvement $(30.0 \%$ of 1884 eligible patients). This was followed by levels $23(8.5 \%)$, level 1 (5.5\%), level 5 (5.3\%), levels 25 (5.3\%), levels 12 (4.0\%), levels 2345 (3.9\%), levels 235 (3.8\%), levels 2rp (3.2\%), levels 234 (3.1\%), level rp (2.4\%), level $3(2.2 \%)$, levels 23rp $(2.1 \%)$, levels $24(1.5 \%)$, and others (19.4\%). Table 3 shows that, taken level 2 as reference, patients with level 1 had an OR of 2.101 (95\% CI: 1.090-4.047, $P=0.027$ ) for distant metastasis, levels 2345 had the highest OR of 4.064 (95\% CI: 2.155-7.666, $P<0.001)$ for distant metastasis, and levels 24 had an OR of 3.003 (95\% CI: 1.074-8.395, $P=0.036$ ) for distant metastasis after adjustment for age, sex and race, which were also statistically significant in univariate analysis.

3.7. Prognostic Values of LN Metastasis Categories for CSS in Patients with NPC. As shown in Table 4, levels 235 had a statistically significant HR of 1.708 (95\% CI: 1.089-2.678, $P=0.020)$ for CCS compared to level 2 after adjustment for age, sex, race, histology, TNM stage, and treatment strategies, while it is not statistically significant before $(P=0.056)$. Level 3 had a significant HR of 1.709 (95\% CI: 1.017-2.873, $P=0.043)$ in univariate analysis; however, it reduced to 1.602 (95\% CI: $0.938-2.737)$ with no statistical significance $(P=0.084)$.

\section{Discussion}

NPC is a malignant tumor of the epithelial tissue, and its incidence and mortality vary by race, sex, age, and country [16]. In our study, the overall U.S. incidence of NPC from 20062015 was 0.33 per 100,000 population. Males are two to three times more likely to develop the disease than women, and the peak age of disease occurrence is between 50 and 60 years [1], which was similar to our results (male-to-female ratio of $2.6: 1$ ). Although substantial declines in NPC incidence rates have been reported in nearly all Asian studies [16], we found that the U.S. incidence remained stable in our study.

In a retrospective cohort study by Challapalli and colleagues, Asian/Pacific patients had the highest NPC incidence rate, but they also had the highest 5-year CSS rate, while American Indian/Alaska Native had the lowest survival [17]. Lee and Ko analyzed epidemiologic patterns and survival rates for NPC patients using the SEER statistical program and found that poor survival was associated with older age, male sex, and KSCC histology [18]. Radiotherapy is the primary and only curative treatment for NPC [19]. A meta-analysis examining the effects of concomitant chemotherapy with and without adjuvant chemotherapy in NPC demonstrated improvement in OS [20]. Sun et al. performed a retrospective population-based analysis and provided evidence of a positive impact of surgery on survival 
TABLE 2: Kaplan-Meier analyses for 5-year OS and CSS rates and multivariate analyses using Cox proportional hazards regression model in all 2994 NPC patients.

\begin{tabular}{|c|c|c|c|c|c|c|c|c|}
\hline \multirow{2}{*}{ Characteristics } & \multicolumn{2}{|c|}{ 5-year OS } & \multicolumn{2}{|c|}{ Cox proportional HR } & \multicolumn{2}{|c|}{ 5-year CSS } & \multicolumn{2}{|c|}{ Cox proportional HR } \\
\hline & Rate (\%) & $P$ & HR (95\% CI) & $P$ & Rate (\%) & $P$ & HR (95\% CI) & $P$ \\
\hline \multicolumn{9}{|l|}{ Age group (years) } \\
\hline$<40$ & 76.6 & $<0.001$ & 1 & & 78.5 & $<0.001$ & 1 & \\
\hline $40-49$ & 67.5 & & $1.394(1.072,1.812)$ & 0.013 & 68.8 & & $1.392(1.057,1.834)$ & 0.019 \\
\hline $50-59$ & 59.9 & & $1.858(1.455,2.373)$ & $<0.001$ & 63.8 & & $1.768(1.366,2.290)$ & $<0.001$ \\
\hline $60-69$ & 49.8 & & $2.422(1.880,3.120)$ & $<0.001$ & 54.4 & & $2.189(1.672,2.866)$ & $<0.001$ \\
\hline$>70$ & 36.1 & & $3.812(2.939,4.945)$ & $<0.001$ & 47.4 & & $3.036(2.289,4.028)$ & $<0.001$ \\
\hline \multicolumn{9}{|l|}{ Sex } \\
\hline Male & 57.6 & 0.002 & 1 & & 61.7 & 0.002 & 1 & \\
\hline Female & 64.1 & & $0.851(0.738,0.980)$ & 0.026 & 68.9 & & $0.843(0.721,0.986)$ & 0.032 \\
\hline \multicolumn{9}{|l|}{ Race $^{\text {a }}$} \\
\hline Asian/Pacific Islander & 67.3 & $<0.001$ & 1 & & 70.2 & $<0.001$ & 1 & \\
\hline White & 55.0 & & $1.476(1.272,1.712)$ & $<0.001$ & 60.2 & & $1.410(1.201,1.657)$ & $<0.001$ \\
\hline Black & 51.8 & & $1.610(1.315,1.971)$ & $<0.001$ & 57.7 & & $1.505(1.209,1.874)$ & $<0.001$ \\
\hline American Indian/Alaska Native & 43.8 & & $1.717(1.117,2.638)$ & 0.014 & 45.3 & & $1.719(1.095,2.698)$ & 0.018 \\
\hline \multicolumn{9}{|l|}{ Histology } \\
\hline KSCC & 48.2 & $<0.001$ & 1 & & 53.8 & $<0.001$ & 1 & \\
\hline NKSCC & 67.9 & & $0.596(0.523,0.679)$ & $<0.001$ & 71.1 & & $0.582(0.505,0.670)$ & $<0.001$ \\
\hline BSCC & 61.8 & & $0.494(0.285,0.858)$ & 0.012 & 66.8 & & $0.491(0.270,0.895)$ & 0.020 \\
\hline \multicolumn{9}{|l|}{ T stage $^{\mathrm{b}}$} \\
\hline $\mathrm{T} 1+\mathrm{T} 2$ & 67.5 & $<0.001$ & 1 & & 72.0 & $<0.001$ & 1 & \\
\hline T3 & 53.4 & & $1.785(1.525,2.089)$ & $<0.001$ & 57.4 & & $1.877(1.581,2.227)$ & $<0.001$ \\
\hline $\mathrm{T} 4$ & 46.5 & & $2.178(1.878,2.527)$ & $<0.001$ & 50.4 & & $2.262(1.924,2.659)$ & $<0.001$ \\
\hline \multicolumn{9}{|l|}{$\bar{N}$ stage $^{c}$} \\
\hline $\mathrm{N} 0+\mathrm{N} 1$ & 62.8 & $<0.001$ & 1 & & 67.7 & $<0.001$ & 1 & \\
\hline $\mathrm{N} 2$ & 57.5 & & $1.311(1.135,1.514)$ & $<0.001$ & 60.9 & & $1.351(1.156,1.580)$ & $<0.001$ \\
\hline $\mathrm{N} 3$ & 47.4 & & $1.625(1.357,1.945)$ & $<0.001$ & 51.1 & & $1.630(1.342,1.979)$ & $<0.001$ \\
\hline \multicolumn{9}{|l|}{ M stage } \\
\hline M0 & 63.5 & $<0.001$ & 1 & & 68.1 & $<0.001$ & 1 & \\
\hline M1 & 23.5 & & $2.888(2.463,3.386)$ & $<0.001$ & 25.5 & & $3.204(2.711,3.786)$ & $<0.001$ \\
\hline \multicolumn{9}{|l|}{ Surgery } \\
\hline Recomme & 65.9 & 0.006 & 1 & & 71.5 & 0.004 & 1 & \\
\hline Not recommended/unknown & 58.2 & & $1.274(1.035,1.568)$ & 0.022 & 62.3 & & $1.261(1.001,1.588)$ & 0.049 \\
\hline \multicolumn{9}{|l|}{ Radiotherapy } \\
\hline Yes & 66.9 & $<0.001$ & 1 & & 71.8 & $<0.001$ & 1 & \\
\hline No & 56.3 & & $1.230(1.044,1.449)$ & 0.013 & 60.4 & & $1.297(1.082,1.554)$ & 0.005 \\
\hline \multicolumn{9}{|l|}{ Chemotherapy } \\
\hline Yes & 62.1 & $<0.001$ & 1 & & 65.9 & $<0.001$ & 1 & \\
\hline No/unknown & 45.6 & & $2.437(2.083,2.850)$ & $<0.001$ & 52.4 & & $2.461(2.071,2.925)$ & $<0.001$ \\
\hline
\end{tabular}

BSCC, basaloid squamous cell carcinoma; CI, confidence interval; CSS, cancer-specific survival; HR, hazard ratio; KSCC, keratinizing squamous cell carcinoma; $\mathrm{M}$, distant metastasis; N, node; NKSCC, nonkeratinizing carcinoma; NPC, nasopharyngeal carcinoma; OS, overall survival; $\mathrm{T}$, tumor. ${ }^{\mathrm{a} T h e}$ 5-year OS and CSS for subjects of unknown race were $73.4 \%$ and $77.7 \%$, respectively. ${ }^{\mathrm{b}} \mathrm{T} 1$, nasopharynx, oropharynx, or nasal cavity; $\mathrm{T} 2$, parapharyngeal extension; $\mathrm{T} 3$, bony structures and/or paranasal sinuses; T4, intracranial extension and/or cranial nerves, hypopharynx, orbit, or infratemporal fossa/masticatory space. ${ }^{\mathrm{C}} \mathrm{N} 0$, none; N1, unilateral cervical and/or unilateral or bilateral retropharyngeal node $(\mathrm{s}), \leq 6 \mathrm{~cm}$ in greatest dimension, above supraclavicular fossa; $\mathrm{N} 2$, bilateral cervical node(s), $\leq 6 \mathrm{~cm}$ in greatest dimension, above supraclavicular fossa; N3, N3a: $>6 \mathrm{~cm}$ in greatest dimension, above supraclavicular fossa; N3b: in supraclavicular fossa.

in NPC [21]. Recent treatment advances have improved clinical outcomes, resulting in longer survival for NPC patients. Our results also show that the 5-year OS was longer for patients that were recommended surgery or received radiotherapy and chemotherapy compared to those that did not receive treatment.

NPC usually presents with LN metastasis, $36 \%-45 \%$ of NPC patients present with the initial symptoms of neck lymphadenectasis, and 60\%-90\% have cervical LN metastasis at NPC diagnosis [22]. A meta-analysis by $\mathrm{Ho}$ et al. revealed that $85 \%$ of 2920 Chinese NPC patients staged using
MRI had LN involvement at diagnosis [23], which is higher than our result $(76.8 \%)$. We found that sex and T stage were not associated with LN metastasis, which is consistent with the report by Tomita and colleagues [24]. They found that LN metastasis was not correlated with sex differences including lifestyle behaviors and biologic traits (e.g., sex hormones). Their study divided patients into two groups ( $\leq 60$ vs. $>60$ years) and found that age was not significantly correlated with the incidence of LN metastasis. However, we stratified age into five groups and found that younger patients ( $<40$ years) had a significantly higher rate of $\mathrm{LN}$ 
TABle 3: Analysis of risk LN metastasis categories for distant metastasis using logistic regression in 1884 eligible NPC patients with LN metastasis.

\begin{tabular}{lccc}
\hline LN metastasis category & $\begin{array}{c}\text { Univariate analysis } \\
\text { OR }(95 \% \text { CI })\end{array}$ & $\begin{array}{c}\text { Multivariate analysis } \\
\text { OR }(95 \% \text { CI })\end{array}$ \\
\hline Level 2 & Reference & Reference \\
Levels 23 & $1.664(0.926,2.990)$ & 0.089 & $1.714(0.948,3.100)$ \\
Level 1 & $2.065(1.079,3.950)$ & 0.029 & $2.101(1.090,4.047)$ \\
Level 5 & $1.622(0.802,3.280)$ & 0.178 & $1.506(0.736,3.084)$ \\
Levels 25 & $1.984(1.019,3.862)$ & 0.044 & $1.949(0.989,3.843)$ \\
Levels 12 & $0.360(0.085,1.519)$ & 0.164 & $0.356(0.084,1.513)$ \\
Levels 2345 & $4.295(2.307,7.999)$ & $<0.001$ & 0.263 \\
Levels 235 & $0.994(0.379,2.608)$ & 0.991 & $0.064(2.155,7.666)$ \\
Levels 2rp & $1.458(0.591,3.596)$ & 0.413 & $1.091(0.413,2.884)$ \\
Levels 234 & $1.215(0.460,3.209)$ & 0.694 & $1.379(0.552,3.449)$ \\
Level rp & $0.938(0.278,3.158)$ & 0.917 & 0.162 \\
Level 3 & $1.382(0.470,4.065)$ & 0.557 & $0.960(0.283,3.253)$ \\
Levels 23rp & $1.930(0.716,5.206)$ & 0.194 & $1.381(0.464,4.110)$ \\
Levels 24 & $2.853(1.030,7.906)$ & 0.044 & $2.103(0.772,5.731)$ \\
\hline
\end{tabular}

CI, confidence interval; LN, lymph node; NPC, nasopharyngeal carcinoma; OR, odds ratio; rp, retropharynx. Level 2 means the patients with only level II LN metastasis. Levels 23 means the patients with level II and level III LN metastases, etc. Multivariate analysis adjusted for age, sex, and race.

TABLE 4: Cox regression analyses for evaluating the risk LN metastasis categories for CSS in 1884 eligible NPC patients with LN metastasis.

\begin{tabular}{|c|c|c|c|c|}
\hline \multirow{2}{*}{ LN metastasis category } & \multicolumn{2}{|c|}{ Univariate analysis } & \multicolumn{2}{|c|}{ Multivariate analysis } \\
\hline & HR $(95 \% \mathrm{CI})$ & $P$ & HR $(95 \%$ CI $)$ & $P$ \\
\hline Level 2 & Reference & & Reference & \\
\hline Levels 23 & $1.106(0.770,1.587)$ & 0.587 & $1.137(0.786,1.646)$ & 0.495 \\
\hline Level 1 & $1.200(0.811,1.776)$ & 0.361 & $1.175(0.790,1.749)$ & 0.426 \\
\hline Level 5 & $1.073(0.704,1.634)$ & 0.745 & $1.048(0.682,1.609)$ & 0.832 \\
\hline Levels 25 & $1.397(0.934,2.090)$ & 0.103 & $1.118(0.736,1.699)$ & 0.600 \\
\hline Levels 12 & $1.361(0.850,2.180)$ & 0.200 & $1.360(0.841,2.201)$ & 0.210 \\
\hline Levels 2345 & $1.338(0.844,2.123)$ & 0.216 & $0.928(0.563,1.528)$ & 0.768 \\
\hline Levels 235 & $1.518(0.989,2.329)$ & 0.056 & $1.708(1.089,2.678)$ & 0.020 \\
\hline Levels 2rp & $0.717(0.365,1.409)$ & 0.334 & $0.588(0.295,1.173)$ & 0.132 \\
\hline Levels 234 & $1.435(0.876,2.349)$ & 0.151 & $1.299(0.772,2.188)$ & 0.325 \\
\hline Level rp & $1.106(0.581,2.105)$ & 0.758 & $0.869(0.452,1.672)$ & 0.674 \\
\hline Level 3 & $1.709(1.017,2.873)$ & 0.043 & $1.602(0.938,2.737)$ & 0.084 \\
\hline Levels 23rp & $1.266(0.665,2.409)$ & 0.472 & $1.248(0.646,2.408)$ & 0.510 \\
\hline Levels $24^{1}$ & $1.889(1.045,3.412)$ & 0.035 & $1.587(0.866,2.908)$ & 0.135 \\
\hline
\end{tabular}

CI, confidence interval; CSS, cancer-specific survival; HR, hazard ratio; LN, lymph node; rp, retropharynx. Level 2 means the patients with only level II LN metastasis, Levels 23 means the patients with level II and III LN metastases, etc. Multivariate analysis adjusted for age, sex, race, histology, TNM stage, and treatment (radiotherapy, surgery, and chemotherapy).

metastasis than older patients. The effect of age on the $\mathrm{LN}$ metastasis remains disputed. In terms of $\mathrm{T}$ stage, $\mathrm{Lv}$ et al. examined 1067 patients with LN involvement in T1-T2 and T3-T4 groups and found no significant correlation between T stage and nodal involvement [25]. This suggests that $\mathrm{T}$ and $\mathrm{N}$ stages are dependent on tumor biological behaviors. Therefore, it is appropriate to make clinical treatment decisions based on the $\mathrm{T}$ and $\mathrm{N}$ stages, rather than the total stage. The WHO pathological type of NPC has different clinical characteristics and prognostic information; it is significantly associated with the incidence of cervical LN metastasis. One retrospective study found that patients with NKSCC showed a higher incidence of LN metastasis than patients with KSCC [24], which was in accordance with our results. Another retrospective population-based analysis reported an LN metastasis rate for BSCC to be $53.7 \%$ [26], which was lower than our rate of $60.5 \%$.
The actual distributions of nodal metastasis as described in terms of LN level differs between studies. Most reported that level II and retropharyngeal LNs were the first affected by metastasis, with overall probabilities of $60 \%-80 \%$ $[23,25]$. Chua et al. found a $29.1 \%$ rate of retropharyngeal LN involvement [27]. Our results showed that the probabilities of level II and retropharyngeal LN involvement were $65.9 \%$ and $13.5 \%$, respectively. These discrepancies may be attributed to variable detection methods and diagnostic standards. Retropharyngeal LN metastases are isodense and contiguous with the primary tumor and thus may not be identified as a separate mass on CT [7]. The superior softtissue contrast of MRI appears to be a better option for LN identification. These factors may have influenced the low incidence of retropharyngeal LN involvement compared to other reports. Several recent studies have reported that LN metastasis in NPC occurs in an orderly fashion from the 
higher to lower level LNs, and skip metastasis is rare, ranging from $0.5 \%$ to $7.9 \%[28,29]$. The overall frequency of skip metastasis in our series was $5.7 \%$.

Metastatic nodes from NPC have been associated with poor outcome, especially for cases with bulky nodes $(>6 \mathrm{~cm})$, necrosis, or nodes in the lower neck $[30,31]$. Compared with the $\mathrm{N} 0+\mathrm{N} 1$ group, the N2 group had a HR of 1.311 (95\% CI: $1.135-1.514, P<0.001)$ for OS and 1.351 for CSS (95\% CI: $1.156-1.580, P<0.001$ ), while the N3 group had a HR of 1.625 (95\% CI: $1.357-1.945, P<0.01)$ for OS and 1.630 for CSS (95\% CI: 1.342-1.979, $P<0.01$ ). Although nodal metastases are associated with poorer prognoses, the recommendations for treatment, the correlation between LN metastases and distant metastases, and how tumor cells enter the bloodstream via LNs still remained debate. Two recent studies in mouse models revealed that distant metastasis is driven by direct invasion of tumor cells into LN blood vessels [32] rather than through the thoracic duct [33]. To examine the influence of LN metastasis on distant metastasis, we classified eligible patients into LN metastasis categories (e.g., level 2, levels 23 , level 1 , level 5, and levels 25).To our knowledge, this is the first population-based analysis to use LN metastasis compound category to provide evidence of the impacts of LN metastasis on distant metastasis and survival in NPC. Taking level 2 as reference, level 1 had an OR of 2.101 (95\% CI: $1.090-4.047, P=0.027$ ) and levels 24 had an OR of 3.003 (95\% CI: $1.074-8.395, P=0.036$ ) for distant metastasis after adjustment for age, sex, and race. Notably, levels 2345 category had the highest OR of 4.064 (95\% CI: 2.155-7.666, $P<0.001)$ for distant metastasis. In survival analysis, levels 235 had a statistically significant HR of 1.708 (95\% CI: $1.089-2.678, P=0.020)$ for CSS compared to level 2 after adjustment for age, sex, race, histology, TNM stage, and treatment strategies, while it was not statistically significant before $(\mathrm{HR}=1.518,95 \%$ CI: 0.989-2.329, $P=0.056)$.

Our results should be interpreted with caution due to limitations associated with use of the SEER database. First, the database does not specify the detection methods or diagnostic criteria of LN involvement. Second, we did not include well-known prognostic factors such as nodal necrosis, nodal extracapsular spread, or laterality. It remains unknown if incorporating these factors would alter the study conclusions. Nevertheless, our findings provide evidence of the impact of LN metastasis category on distant metastasis and survival in NPC.

\section{Conclusions}

The incidence of LN involvement in NPC patients differed by age, race, histologic type, and $M$ stage but not sex or $\mathrm{T}$ stage. Taking level 2 as reference, level 1, levels 2345, and levels 24 categories had significantly higher risks for distant metastasis after adjustment for age, sex, and race. Levels 235 had a statistically significant HR of 1.708 for CSS compared to level 2 after adjustment for age, sex, race, histology, TNM stage, and treatment.

\section{Data Availability}

All data were acquired from the Surveillance, Epidemiology, and End Results (SEER) database. The data are available from the corresponding author upon request.

\section{Conflicts of Interest}

The authors declare that there are no conflicts of interest regarding the publication of this paper.

\section{Authors' Contributions}

Yali $\mathrm{Xu}$ and Taoyuan Huang contributed equally to this work.

\section{Acknowledgments}

The authors acknowledge the efforts of the Surveillance, Epidemiology, and End Results (SEER) Program tumor registries in the creation of the SEER database.

\section{References}

[1] J. Ferlay, I. Soerjomataram, R. Dikshit et al., "Cancer incidence and mortality worldwide: sources, methods and major patterns in GLOBOCAN 2012," International Journal of Cancer, vol. 136, no. 5, pp. E359-E386, 2015.

[2] G. Zada, M. B. S. Lopes, S. Mukundan, and E. R. Laws, "Nasopharyngeal carcinoma and squamous cell carcinoma of the paranasal sinuses," Atlas of Sellar and Parasellar Lesions, Springer International Publishing, Cham, Switzerland, 2016.

[3] H. Y. Wang, Y. L. Chang, K. F. To et al., "A new prognostic histopathologic classification of nasopharyngeal carcinoma," Chinese Journal of Cancer, vol. 35, no. 1, p. 41, 2016.

[4] J. S. T. Sham, D. Choy, and W. I. Wei, "Nasopharyngeal carcinoma: orderly neck node spread," International Journal of Radiation Oncology*Biology*Physics, vol. 19, no. 4, pp. 929-933, 1990.

[5] Y. Xu, X. Chen, M. Zhang et al., "Prognostic effect of parotid area lymph node metastases after preliminary diagnosis of nasopharyngeal carcinoma: a propensity score matching study," Cancer Medicine, vol. 6, no. 10, pp. 2213-2221, 2017.

[6] Y. Feng, C. Cao, Q. Hu, and X. Chen, "Prognostic value and staging classification of lymph nodal necrosis in nasopharyngeal carcinoma after intensity-modulated radiotherapy," Cancer Research and Treatment, vol. 51, no. 3, pp. 1222-1230, 2019.

[7] L.-Z. Liu, G.-Y. Zhang, C.-M. Xie, X.-W. Liu, C.-Y. Cui, and L. Li, "Magnetic resonance imaging of retropharyngeal lymph node metastasis in nasopharyngeal carcinoma: patterns of spread," International Journal of Radiation Oncology*Biology*Physics, vol. 66, no. 3, pp. 721-730, 2006.

[8] L. Tang, L. Li, Y. Mao et al., "Retropharyngeal lymph node metastasis in nasopharyngeal carcinoma detected by magnetic resonance imaging: prognostic value and staging categories," Cancer, vol. 113, no. 2, pp. 347-354, 2010.

[9] X. Wang, C. Hu, H. Ying et al., "Patterns of lymph node metastasis from nasopharyngeal carcinoma based on the 2013 updated consensus guidelines for neck node levels," Radiotherapy and Oncology, vol. 115, no. 1, pp. 41-45, 2015.

[10] K. Saarilahti, M. Kouri, J. Collan et al., "Intensity modulated radiotherapy for head and neck cancer: evidence for preserved 
salivary gland function," Radiotherapy and Oncology, vol. 74, no. 3, pp. 251-258, 2005.

[11] T.-L. Huang, C.-Y. Chien, W.-L. Tsai et al., "Long-term late toxicities and quality of life for survivors of nasopharyngeal carcinoma treated with intensity-modulated radiotherapy versus non-intensity-modulated radiotherapy," Head \& Neck, vol. 38, no. S1, pp. E1026-E1032, 2016.

[12] N. Lee, J. Harris, A. S. Garden et al., "Intensity-modulated radiation therapy with or without chemotherapy for nasopharyngeal carcinoma: radiation therapy oncology group phase II trial 0225," Journal of Clinical Oncology, vol. 27, no. 22, pp. 3684-3690, 2009.

[13] J. Zong, J. Lin, C. Hu et al., "Nasopharyngeal carcinoma treated with intensity modulated radiation therapy using a reduced clinical target volume for the neck nodal regions," International Journal of Radiation Oncolog $y *$ Biology*Physics, vol. 96, no. 2, pp. E343-E344, 2016.

[14] B. F. Hankey, L. A. Ries, and B. K. Edwards, "The surveillance, epidemiology, and end results program: a national resource," Cancer Epidemiology, Biomarkers \& Prevention, vol. 8, no. 12, pp. 1117-1121, 1999.

[15] Y. Ren, H. Qiu, Y. Yuan et al., "Evaluation of 7th edition of AJCC staging system for nasopharyngeal carcinoma," Journal of Cancer, vol. 8, no. 9, pp. 1665-1672, 2017.

[16] K.-R. Wei, R.-S. Zheng, S.-W. Zhang, Z.-H. Liang, Z.-M. Li, and W.-Q. Chen, "Nasopharyngeal carcinoma incidence and mortality in China, 2013," Chinese Journal of Cancer, vol. 36, no. 1, p. 90, 2017.

[17] S. D. Challapalli, M. C. Simpson, E. Adjei Boakye et al., "Survival differences in nasopharyngeal carcinoma among racial and ethnic minority groups in the United States: a retrospective cohort study," Clinical Otolaryngology, vol. 44, no. 1, pp. 14-20, 2019.

[18] J. T. Lee and C. Y. Ko, "Has survival improved for nasopharyngeal carcinoma in the United States?," OtolaryngologyHead and Neck Surgery, vol. 132, no. 2, pp. 303-308, 2005.

[19] M. L. K. Chua, J. T. S. Wee, E. P. Hui, and A. T. C. Chan, "Nasopharyngeal carcinoma," The Lancet, vol. 387, no. 10022, pp. 1012-1024, 2016.

[20] P. Blanchard, A. Lee, S. Marguet et al., "Chemotherapy and radiotherapy in nasopharyngeal carcinoma: an update of the MAC-NPC meta-analysis," The Lancet Oncology, vol. 16, no. 6, pp. 645-655, 2015.

[21] J. Sun, Z. Huang, Z. Hu, and R. Sun, "Benefits of local tumor excision and pharyngectomy on the survival of nasopharyngeal carcinoma patients: a retrospective observational study based on SEER database," Journal of Translational Medicine, vol. 15, no. 1, p. 116, 2017.

[22] W. I. Wei and J. S. Sham, "Nasopharyngeal carcinoma," The Lancet, vol. 365, no. 9476, pp. 2041-2054, 2005.

[23] F. C. Ho, I. W. Tham, A. Earnest, K. M. Lee, and J. J. Lu, "Patterns of regional lymph node metastasis of nasopharyngeal carcinoma: a meta-analysis of clinical evidence," BMC Cancer, vol. 12, no. 1, p. 98, 2012.

[24] N. Tomita, N. Fuwa, Y. Ariji, T. Kodaira, and N. Mizoguchi, "Factors associated with nodal metastasis in nasopharyngeal cancer: an approach to reduce the radiation field in selected patients," The British Journal of Radiology, vol. 84, no. 999, pp. 265-270, 2011.

[25] J. Lv, R. Wang, Y. Qing, Q. Du, and T. Zhang, "Magnetic resonance imaging analysis of regional lymph node metastasis in 1298 cases of nasopharyngeal carcinoma," Lin Chung Er Bi Yan Hou Tou Jing Wai Ke Za Zhi, vol. 26, no. 18, pp. 769-772, 2012.
[26] A. A. Unasl, J. R. Booth, N. A. Rossi, J. K. Byrd, and S. E. Kountakis, "Basaloid nasopharyngeal carcinoma: a population-based analysis of a rare tumor," The Laryngoscope, vol. 10, 2019.

[27] D. T. T. Chua, J. S. T. Sham, D. L. W. Kwong, G. K. H. Au, and D. T. K. Choy, "Retropharyngeal lymphadenopathy in patients with nasopharyngeal carcinoma: a computed tomography-based study," Cancer, vol. 79, no. 5, pp. 869-877, 1997.

[28] L. Tang, Y. Mao, L. Liu et al., "The volume to be irradiated during selective neck irradiation in nasopharyngeal carcinoma: analysis of the spread patterns in lymph nodes by magnetic resonance imaging," Cancer, vol. 115, no. 3, pp. 680-688, 2009.

[29] S. H. Ng, J. T. Chang, S. C. Chan et al., "Nodal metastases of nasopharyngeal carcinoma: patterns of disease on MRI and FDG PET," European Journal of Nuclear Medicine and Molecular Imaging, vol. 31, no. 8, pp. 1073-1080, 2004.

[30] J. J. Pan, W. T. Ng, J. F. Zong et al., "Proposal for the 8th edition of the AJCC/UICC staging system for nasopharyngeal cancer in the era of intensity-modulated radiotherapy," Cancer, vol. 122, no. 4, pp. 546-558, 2016.

[31] Q.-Y. Ai, A. D. King, D. M. C. Poon et al., "Extranodal extension is a criterion for poor outcome in patients with metastatic nodes from cancer of the nasopharynx," Oral Oncology, vol. 88, pp. 124-130, 2019.

[32] E. R. Pereira, D. Kedrin, G. Seano et al., "Lymph node metastases can invade local blood vessels, exit the node, and colonize distant organs in mice," Science, vol. 359, no. 6382, pp. 1403-1407, 2018.

[33] M. Brown, F. P. Assen, A. Leithner et al., "Lymph node blood vessels provide exit routes for metastatic tumor cell dissemination in mice," Science, vol. 359, no. 6382, pp. 14081411, 2018. 


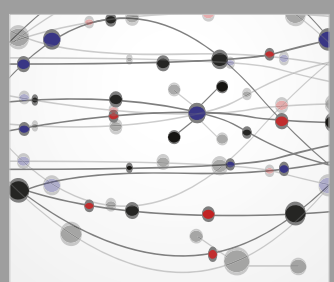

The Scientific World Journal
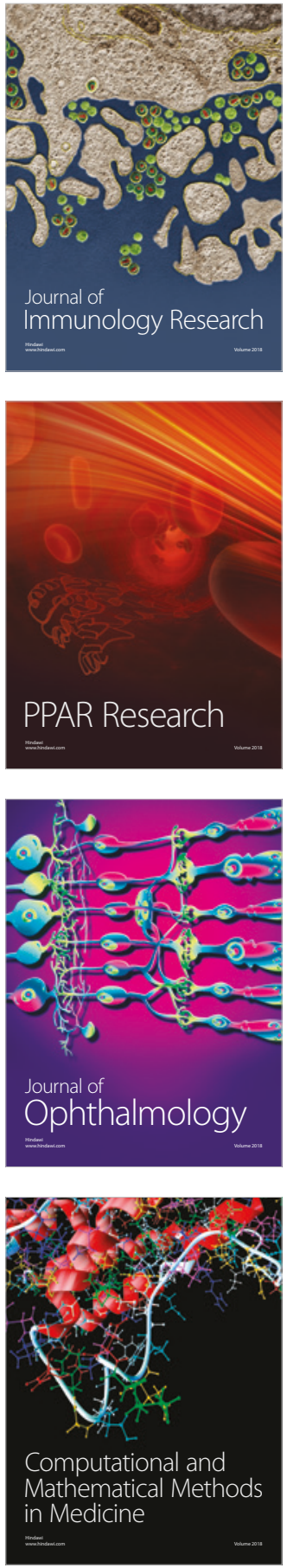

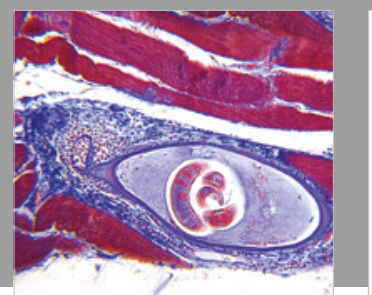

Gastroenterology Research and Practice

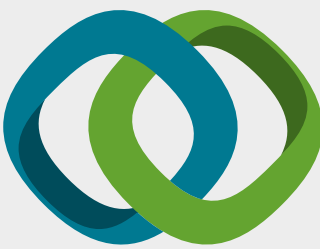

\section{Hindawi}

Submit your manuscripts at

www.hindawi.com
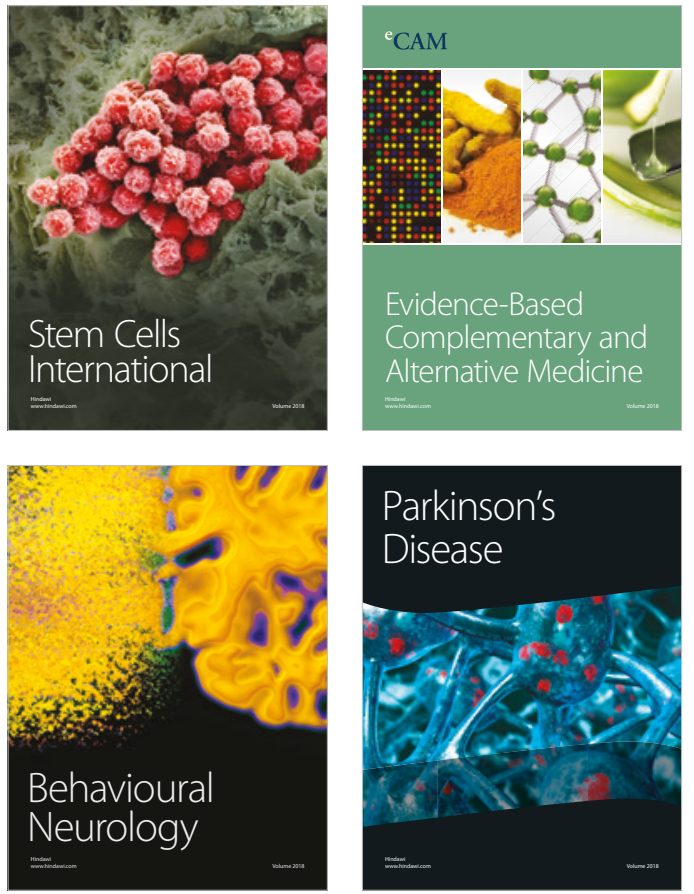

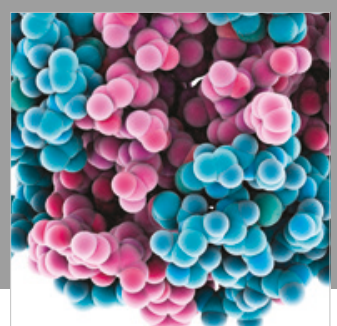

ournal of

Diabetes Research

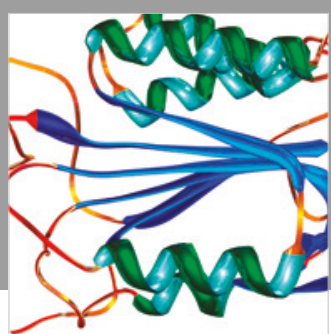

Disease Markers
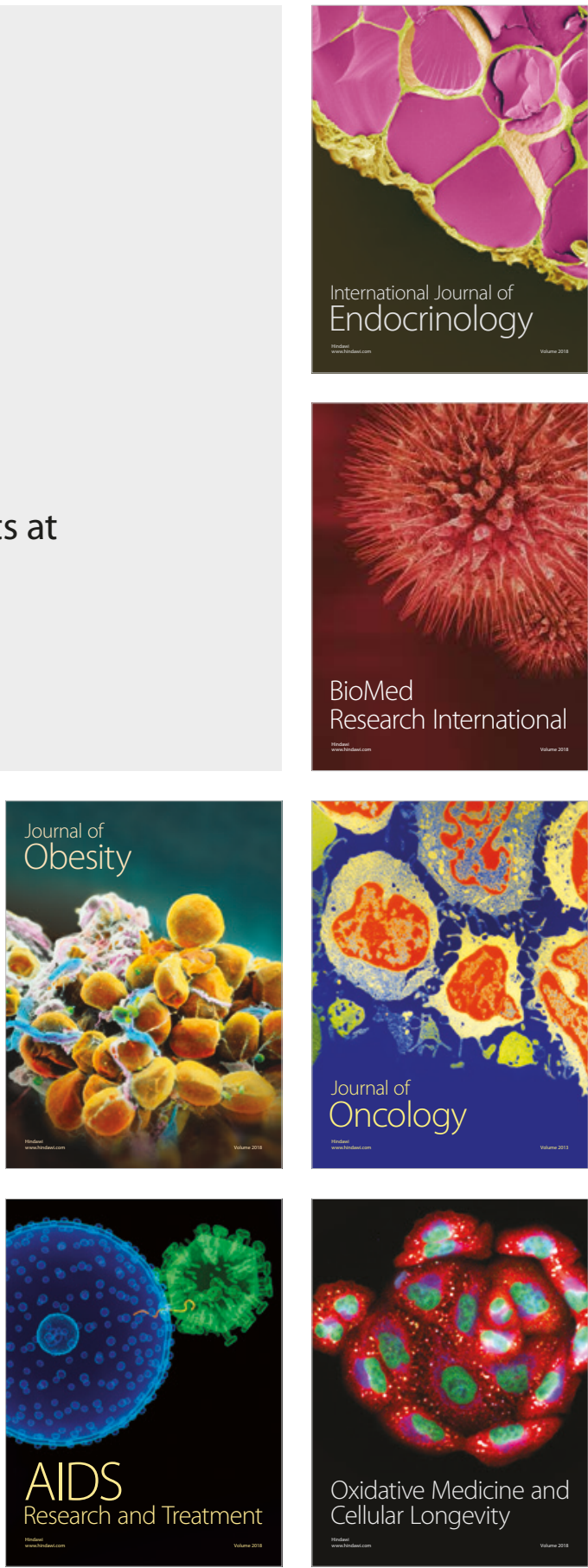